induced psoriasis-like skin inflammation. J. Clin. Invest. 116:2094-2104. doi:10.1172/JCI27179.

13. Djalilian, A.R., et al. 2006. Connexin 26 regulates epidermal barrier and wound remodeling and promotes psoriasiform response. J. Clin. Invest. 116:1243-1253. doi:10.1172/JCI27186.

14. Gillitzer, R., et al. 1993. MCP-1 mRNA expression in basal keratinocytes of psoriatic lesions. J. Invest. Dermatol. 101:127-131.

15. van den Oord, J.J., and de Wolf-Peeters, C. 1994. Epithelium-lining macrophages in psoriasis. Br. J. Dermatol. 130:589-594.

16. Djemadji-Oudjiel, N., Goerdt, S., Kodelja, V., Schmuth, M., and Orfanos, C.E. 1996. Immunohistochemical identification of type II alternatively activated dendritic macrophages (RM $3 / 1+3$, MS-1+/-, 25F9-) in psoriatic dermis. Arch. Dermatol. Res. 288:757-764.

17. Vestergaard, C., et al. 2004. Expression of CCR2 on monocytes and macrophages in chronically inflamed skin in atopic dermatitis and psoriasis. Acta Derm. Venereol. 84:353-358.

18. Boehncke, W.H., Wortmann, S., Kaufmann, R. Mielke, V., and Sterry, W. 1995. A subset of mac- rophages located along the basement membrane ("lining cells") is a characteristic histopathological feature of psoriasis. Am. J. Dermatopathol. 17:139-144.

19. Nickoloff, B.J. 2000. Characterization of lymphocyte-dependent angiogenesis using a SCID mouse: human skin model of psoriasis. J. Investig. Dermatol. Symp. Proc. 5:67-73

20. Wang, H., et al. 2006. Activated macrophages are essential in a murine model for $\mathrm{T}$ cell-mediated chronic psoriasiform skin inflammation. J. Clin. Invest. 116:2105-2114. doi:10.1172/JCI27180.

21. Schlaak, J.F., et al. 1994. T cells involved in psoriasis vulgaris belong to the Th1 subset. J. Invest. Dermatol. 102:145-149.

22. Ackermann, L., and Harvima, I.T. 1998. Mast cells of psoriatic and atopic dermatitis skin are positive for TNF-alpha and their degranulation is associated with expression of ICAM-1 in the epidermis. Arch. Dermatol. Res. 290:353-359.

23. Gottlieb, A.B., et al. 2003. A randomized trial of etanercept as monotherapy for psoriasis. Arch. Dermatol. 139:1627-1632; discussion 1632.

24. Leonardi, C.L., et al. 2003. Etanercept as mono- therapy in patients with psoriasis. N. Engl. J. Med. 349:2014-2022.

25. Kess, D., et al. 2003. CD4+ T cell-associated pathophysiology critically depends on CD18 gene dose effects in a murine model of psoriasis. J. Immunol. 171:5697-5706.

26. Paukkonen, K., Naukkarinen, A., and Horsmanheimo, M. 1992. The development of manifest psoriatic lesions is linked with the invasion of CD8 $+\mathrm{T}$ cells and CD11c + macrophages into the epidermis. Arch. Dermatol. Res. 284:375-379.

27. Banchereau, J., and Steinman, R.M. 1998. Dendritic cells and the control of immunity. Nature. 392:245-252.

28. Janeway, C.A., Jr., and Medzhitov, R. 2002. Innate immune recognition. Annu. Rev. Immunol. 20:197-216.

29. Nestle, F.O., et al. 2005. Plasmacytoid predendritic cells initiate psoriasis through interferon-alpha production. J. Exp. Med. 202:135-143.

30. Nestle, F.O., Turka, L.A., and Nickoloff, B.J. 1994. Characterization of dermal dendritic cells in psoriasis. Autostimulation of T lymphocytes and induction of Th1 type cytokines. J. Clin. Invest. 94:202-209.

\title{
Tuning the oviduct to the anandamide tone
}

\author{
Herbert Schuel
}

Division of Anatomy and Cell Biology, Department of Pathology and Anatomical Sciences, School of Medicine and Biomedical Sciences, University at Buffalo, State University of New York, Buffalo, New York, USA.

\begin{abstract}
Anandamide ( $N$-arachidonoylethanolamide) is a lipid signal molecule that was the first endogenous agonist for cannabinoid receptors to be discovered. Cannabinoid receptor type 1 (CB1) is widely distributed in neurons and nonneuronal cells in brain and peripheral organs including sperm, eggs, and preimplantation embryos. A study by Wang and colleagues in this issue of the JCI demonstrates that a critical balance between anandamide synthesis by $N$-acylphosphatidylethanolamine-selective phospholipase D (NAPE-PLD) and its degradation by fatty acid amide hydrolase (FAAH) in mouse embryos and oviducts creates locally an appropriate "anandamide tone" required for normal embryo development, oviductal transport, implantation, and pregnancy (see the related article beginning on page 2122). Adverse effects of elevated levels of anandamide on these processes resulting from FAAH inactivation are mimicked by administration of (-)- $\Delta^{9}$-tetrahydrocannabinol (THC; the major psychoactive constituent of marijuana), due to enhanced signaling via CB1. These findings show that exogenous THC can swamp endogenous anandamide signaling systems, thereby affecting multiple physiological processes.
\end{abstract}

Attempts to understand the mechanisms responsible for the psychoactive properties of tetrahydrocannabinol (THC) in marijuana led to the discovery of canna-

Nonstandard abbreviations used: 2-AG, 2-arachidonoylglycerol; CB1, cannabinoid receptor type 1; FAAH, fatty acid amide hydrolase; NAPE-PLD, $N$-acylphosphatidylethanolamine-selective phospholipase D; THC, $(-)-\Delta^{9}$-tetrahydrocannabinol.

Conflict of interest: The author has declared that no conflict of interest exists.

Citation for this article: J. Clin. Invest. 116:2087-2090 (2006). doi:10.1172/JCI29424. binoid receptors and their endogenous ligands, the endocannabinoids (1). Cannabinoid receptors are $G$ protein-coupled receptors. Two subtypes have been cloned and characterized to date. Cannabinoid receptor type 1 (CB1) was originally cloned from rat and human brain, while CB2 was originally cloned from human promyelocytic leukemia HL-60 cells. Anandamide and related fatty acid derivatives that activate these receptors are endocannabinoids. Anandamide is released from membrane phospholipid precursors by
$N$-acylphosphatidylethanolamine-selective phospholipase D (NAPE-PLD) when neurons and other cells are stimulated by neurotransmitters and hormones. Released anandamide is quickly eliminated by membrane-bound fatty acid amide hydrolase (FAAH), indicative of a role for this lipid in cell signaling.

Anandamide signaling via cannabinoid receptors is not restricted to the central nervous system. We now know that CB1, CB2, NAPE-PLD, and FAAH are widely distributed in nonneuronal somatic cells of peripheral organs including those of the reproductive system, as well as in germ cells of vertebrates and invertebrates (1-8). This may account for the effects of marijuana and THC on multiple aspects of reproductive physiology, including secretion of gonadotrophic hormones by the pituitary gland, secretion of sex steroids by gonads, sperm production, ovulation, mating behavior, sperm capacitation, fertilization, early embryonic development, implantation of blastocysts into the uterine endometrium, placental functions, fetal growth, number of pregnancies carried to term, lactation, suckling behavior of newborns, postnatal development, and growth of malignant breast and prostate cells. 


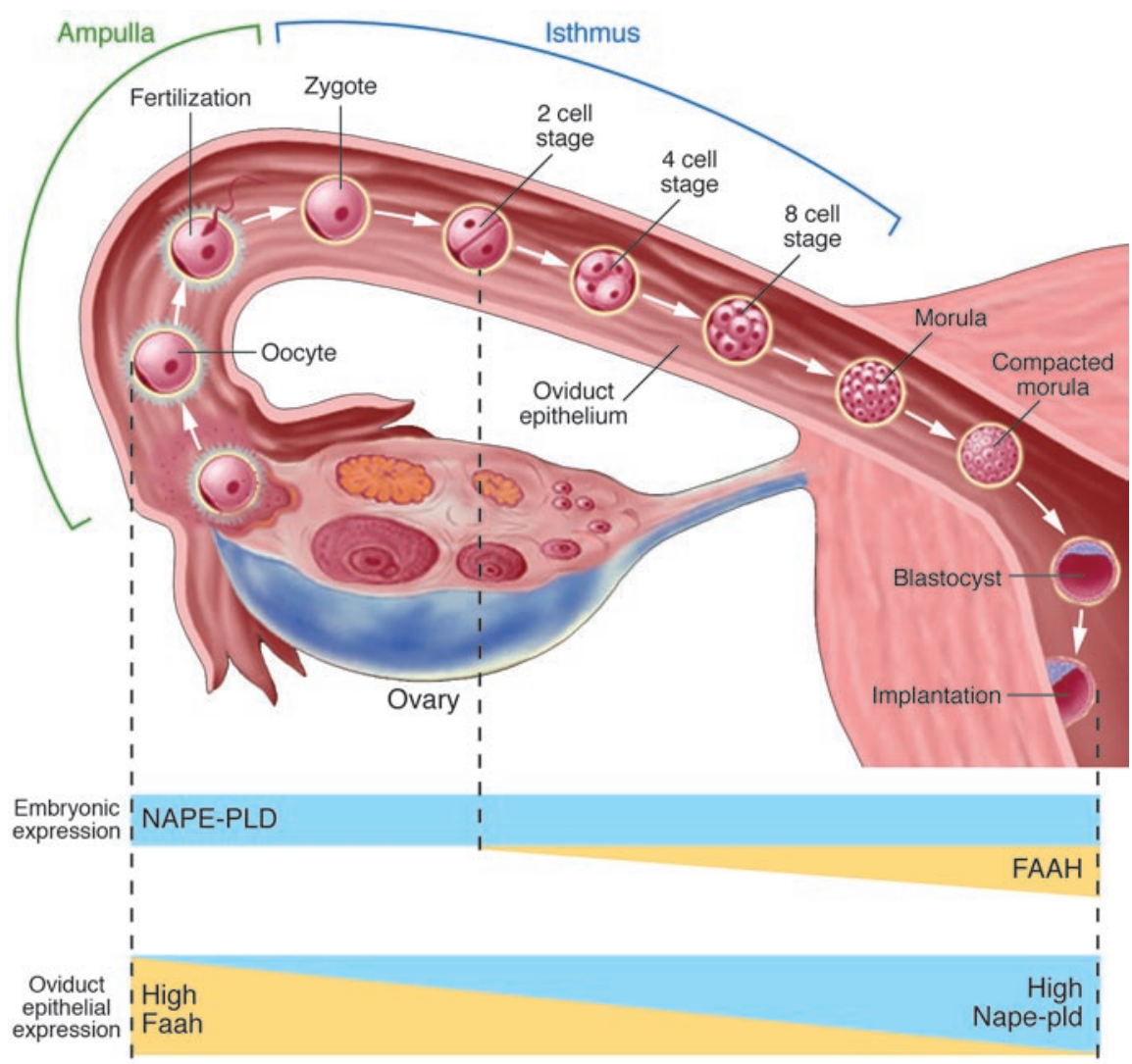

\section{Figure 1}

FAAH is a key metabolic gatekeeper regulating anandamide tone in oviduct and preimplantation embryos. CB1, NAPE-PLD, and FAAH are expressed in the oviduct and embryo. A concentration gradient for anandamide in the oviduct is suggested by the expression of higher levels of Nape-pld in the oviduct epithelium of the isthmus, in contrast to higher levels of Faah in the ampulla (9). Elevating anandamide levels in the oviduct by administration of FAAH inhibitors or by mating $\mathrm{Faah}^{-/}$mice inhibits cleavage, impairs oviductal transport of preimplantation embryos, and reduces fertility. NAPE-PLD was detected in mouse embryos at the stage of the fertilized egg through to the blastocyst stage, while FAAH first appeared in 2-cell embryos. Treatment of mice with THC causes retarded development of embryos and oviductal retention of embryos. The adverse effects of inactivating FAAH and of administering exogenous THC on these processes are prevented by administration of the CB1-selective antagonist SR141716, which suggests that these effects are mediated via CB1. Exposure to $\mathrm{THC}$ swamps the endogenous anandamide tone, leading to early pregnancy failure. Figure adapted from an image kindly provided by S.S. Gambhir and J. Strommer, Stanford University, Stanford, California, USA.

\section{FAAH is the metabolic gatekeeper for anandamide signaling}

Endocannabinoid signaling via CB1 regulates cleavage of mouse eggs, oviductal transport of preimplantation embryos, blastocyst hatching from the zona pellucida, and implantation of blastocysts into the uterine mucosa $(3,6,7)$. In the study by Wang et al. in this issue of the JCI (9), NAPE-PLD was detected in mouse embryos at the stage of the fertilized egg through to the blastocyst stage, while FAAH first appeared in 2-cell embryos (Figure 1). FAAH expression was upregulated in the morula and blastocyst. FAAH was mostly expressed in trophectoderm of blastocysts, suggesting an active role of embryonic FAAH in reducing anandamide levels at potential implantation sites in the uterine mucosa $(6,9)$. An inverse distribution of these enzymes was present in mouse oviducts on days 1-4 of pregnancy, with higher levels of Nape-pld in epithelium of the isthmus than of the ampulla (9). In contrast, Faah was expressed at higher levels in ampulla than in isthmus epithelium. Suppression of FAAH activity in embryos and oviducts pharmacologically or by genetic ablation elevated anandamide lev- els in situ, which resulted in inhibition of embryonic development and expression of cell lineage genes required for differentiation, retention of embryos within the oviduct, impaired implantation, and reduced fertility. Administration of exogenous THC similarly affected early pregnancy events. Furthermore, the adverse effects on initiation of pregnancy resulting from inactivation of FAAH and by addition of THC were blocked by the CB1 selective antagonist SR141716. Unlike endogenous neurotransmitters/neuromodulators, which are rapidly released and degraded locally on demand in the body, exogenous drugs such as THC persist and swamp endogenous anandamide signaling systems, thereby exerting long-term effects on multiple physiological processes. The findings reported by Wang et al. (9) describe an extremely provocative model for the biological effects of THC and marijuana on functions of other peripheral organs as well as the brain.

\section{Clinical implications}

Aberrant functioning of anandamide signaling systems in embryos and oviducts in women may lead to ectopic pregnancy in the oviduct and/or impaired fertility (3,
$7,9)$. Similar adverse effects may be associated with abuse of marijuana by women of reproductive age. This possibility is consistent with studies showing that women who smoke tobacco show an increased incidence of ectopic pregnancy (10), which correlates with the direct inhibitory effects of tobacco smoke and nicotine on oviductal transport of embryos in hamsters (11). Faab-null female mice are less fertile than wild-type females (9). This observation is clinically relevant, since reduced peripheral FAAH activity is associated with spontaneous abortion in women (4). Oviductal transport of embryos in mice was shown to be regulated by crosstalk between anandamide signaling via CB1 and $\beta$-adrenergic receptors in the oviductal muscularis (7). Acetaminophen is a popular over-the-counter analgesic and antiinflammatory agent. Following deacylation, it is converted in the body to the bioactive $N$-acylphenolamine (also known as AM404) via FAAHdependent arachidonic acid conjugation (12). AM404 is a ligand for CB1 receptors and is a potent inhibitor of anandamide reuptake, the inhibition of which leads to elevated anandamide levels in the body. Do women using $\beta$-adrenergic medications or 
acetaminophen show elevated incidences of ectopic pregnancy and impaired fertility?

There are provocative similarities between anandamide signaling in embryos and oviductal epithelia and signaling pathways associated with sperm capacitation in the female reproductive tract. Studies on human and porcine sperm suggested the possible existence of an anandamide concentration gradient in the oviduct that regulates sperm capacitation $(2,3,8,9,13$, 14). A mechanism underlyng this postulated anandamide gradient is indicated by the differences in the distribution of Nape-pld and Faah in mouse oviduct (9). Sperm and preimplantation mouse embryos express functional CB1 receptors as well as key enzymes (NAPE-PLD and FAAH) for the release of anandamide and its subsequent rapid removal $(3,6,8,9,13,14)$. Anandamide is present in human mid-cycle oviductal fluid $(2,3,8,14)$. With respect to mouse embryos, maternal NAPE-PLD and FAAH in the oviduct are primarily concerned with regulation of oviductal transport, while in the embryo these proteins regulate embryonic development (9). Does a similar division of labor regulate sperm capacitation and fertilizing potential within the oviduct?

As the first endocannabinoid to be discovered, anandamide has attracted the most attention from investigators (1). Similarly, the biological functions of CB1 have been studied much more extensively than those mediated by CB2, because CB1 receptors in the brain are directly involved in the psychoactive effects of THC. Significantly, porcine sperm and preimplantation mouse embryos express CB2 receptors (6, $8,13)$. The function(s) of CB2 in sperm and embryos have yet to be examined. We also do not know whether other endocannabinoids (e.g., ethanolamides of other fatty acids, 2-arachidonoylglycerol [2-AG], noladin ether, virodhamine, etc.) are constituents of reproductive tract fluids. If so, how do these endocannabinoids affect sperm capacitation and fertilizing potential, as well as embryo development and transport? Anandamide mimics the effects of THC by serving as a partial agonist for CB1 and CB2 (15). Furthermore, 2-AG is found at much higher concentrations than anandamide in the brain and peripheral organs. 2-AG is a full agonist for both CB1 and CB2. Thus, 2-AG may be a more physiologically significant agonist for cannabinoid receptors than anandamide. Studies on neurons and other somatic cells suggest that endocannabinoids may elicit different responses within the same cell population by binding to different receptor subtypes or splice variants, differentially colocalizing receptors with signaling molecules, and differentially activating $G$ proteins $(16,17)$. Hence, the presence of other endocannabinoids in the microenvironment surrounding sperm or preimplantation embryos might orchestrate subtle differences in their physiology.

\section{Non-CB1 and non-CB2 receptors for anandamide}

Anandamide also can produce biological responses via mechanisms that do not involve cannabinoid receptors $(8,17)$. It is an agonist for transient receptor potential channel vanilloid receptor subunit 1 (TRPV1) and can directly inhibit certain $\mathrm{Ca}^{2+}$ and $\mathrm{K}^{+}$channels, directly activate glutamate ionotropic receptors, and directly block activation of nicotinic $\alpha$ polypeptide 7 receptor by acetylcholine. Significantly, anandamide inhibits spontaneous acrosome reactions in boar sperm during capacitation by activating TRPV1 receptors (13). Thus, alternate pathways for the actions of anandamide and other endocannabinoids in reproductive physiology and other bodily functions warrant careful examination.

\section{Drug development}

Anandamide signaling is directly involved in a myriad of physiological and pathological processes and presents many potential targets for the development of novel therapeutic drugs (18). Unfortunately, cannabinergic ligands affect almost every physiological system investigated (1). Thus, drugs acting on anandamide signaling may produce a wide variety of side effects that would limit their utility. For example, possible medicinal uses of THC are limited by its psychoactive properties. One strategy for drug development targets specific cannabinoid receptors. The CB1 antagonist SR141716 (rimonabant; trade name Acomplia) suppresses appetite (19). The European Medicines Agency recently recommended approval of Acomplia for use as a weight-loss drug. An alternate approach targets removal and degradation of anandamide (18). Basal levels of anandamide in the brain and peripheral organs are quite low (15). It is rapidly released and degraded locally. Thus, drugs that prevent anandamide reuptake and hydrolysis might be useful clinically (18). Given the results pre- sented in the current report (9), such drugs may need to be carefully evaluated to judge their effects on women of reproductive age and those that are pregnant.

Address correspondence to: Herbert Schuel, Division of Anatomy and Cell Biology, Department of Pathology and Anatomical Sciences, School of Medicine and Biomedical Sciences, 206 Farber Hall, University at Buffalo, SUNY, Buffalo, New York 14214, USA. Phone: (716) 829-3578; Fax: (716) 8292911; E-mail: schuel@acsu.buffalo.edu.

1. Onaivi, E.S., Sugiura, T., and Di Marzo, V., editors. 2006. Endocannabinoids: the brain and body's marijuana and beyond. CRC Press/Taylor \& Francis Group. London, United Kingdom. 563 pp.

2. Schuel, H., et al. 2002. $N$-acylethanolamines in human reproductive fluids. Chem. Phys. Lipids. 121:211-227.

3. Schuel, H., and Burkman, L.J. 2006. Endocannabinoids in fertilization, pregnancy and development. In Endocannabinoids: the brain and body's marijuana and beyond. E.S. Onaivi, T. Sugiura, and V. Di Marzo, editors. CRC Press/Taylor \& Francis Group. London, United Kingdom. 467-513.

4. Maccarrone, M., and Finazzi-Agro, A. 2004. Anandamide hydrolase: a guardian angel of human reproduction? Trends Pharmacol. Sci. 25:353-357.

5. Park, B., McPartland, J.M., and Glass, M. 2004. Cannabis, cannabinoids and reproduction. Prostaglandins Leukot. Essent. Fatty Acids. 70:189-197.

6. Paria, B.C., Wang, H., and Dey, S.K. 2002. Endocannabinoid-signaling in synchronizing embryo development and uterine receptivity for implantation. Chem. Phys. Lipids. 121:201-210.

7. Wang, H., et al. 2004. Aberrant cannabinoid signaling impairs oviductal transport of embryos. Nat. Med. 10:1074-1080.

8. Schuel, H., and Burkman, L.J. 2005. A tale of two cells: endocannabinoid-signaling regulates functions of neurons and sperm. Biol. Reprod. 73:1078-1086.

9. Wang, H., et al. 2006. Fatty acid amide hydrolase deficiency limits early pregnancy events. J. Clin. Invest. 116:2122-2131. doi:10.1172/JCI28621.

10. Stillman, R.J., Rosenberg, M.J., and Sachs, B.P. 1986. Smoking and reproduction. Fertil. Steril. 46:545-566.

11. Di Carlantonio, G., and Talbot, P. 1999. Inhalation of mainstream and sidestream cigarette smoke retards embryo transport and slows muscle contraction in oviducts of hamsters (Mesocricetus auratus). Biol. Reprod. 61:651-656.

12. Hogestatt, E.D., et al. 2005. Conversion of acetaminophen to the bioactive $N$-acylphenolamine AM404 via fatty acid amide hydrolase-dependent arachidonic acid conjugation in the nervous system. J. Biol. Chem. 280:31405-31412.

13. Maccarrone, M., et al. 2005. Characterization of the endocannabinoid system in boar spermatozoa and implications for sperm capacitation and acrosome reaction. J. Cell Sci. 118:4393-4404.

14. Schuel, H., et al. 2002. Evidence that anandamide-signaling regulates human sperm functions required for fertilization. Mol. Reprod. Dev. 63:376-387.

15. Sugiura, T., Oka, S., Ikeda, S., and Waku, K. 2006. Occurrence, biosynthesis and metabolism of endocannabinoids. In Endocannabinoids: the brain and body's marijuana and beyond. E.S. Onaivi, T. Sugiura, and V. Di Marzo, editors. CRC Press/Taylor \& Francis Group. London, United Kingdom. 177-214.

16. McAllister, S.D., and Abood, M.E. 2006. Endocan- 
nabinoids and intracellular signaling. In Endocannabinoids: the brain and body's marijuana and beyond. E.S. Onaivi, T. Sugiura, and V. Di Marzo, editors. CRC Press/Taylor \& Francis Group, London, United Kingdom. 119-131.

17. Di Marzo, V., and De Petrocellis, L. 2006. Non$\mathrm{CB} 1$, non-CB2 receptors for endocannabinoids. In
Endocannabinoids: the brain and body's marijuana and beyond. E.S. Onaivi, T. Sugiura, and V. Di Marzo, editors. CRC Press/Taylor \& Francis Group. London, United Kingdom. 151-174.

18. Ligresti, A., and Di Marzo, V. 2006. Endocannabinoid-based molecules as potential therapeutic drugs. In Endocannabinoids: the brain and body's mari- juana and beyond. E.S. Onaivi, T. Sugiura, and V. Di Marzo, editors. CRC Press/Taylor \& Francis Group. London, United Kingdom. 537-554.

19. Despres, J.P., Golay, A., and Sjostrom, L. 2005. Effects of rimonabant on metabolic risk factors in overweight patients with dyslipidemia. N. Engl. J. Med. 353:2121-2134.

\section{The glomerular basement membrane: not gone, just forgotten}

Marilyn G. Farquhar

Department of Cellular and Molecular Medicine, UCSD, La Jolla, California, USA.

\begin{abstract}
The glomerular capillaries function as the filtration barrier that retains albumin and other plasma proteins in the circulation. The unresolved question that has been asked for more than 50 years is, Which structural component of these capillaries constitutes the main molecular sieve that normally retains albumin and allows its passage in diseases associated with proteinuria? There is considerable evidence implicating both the glomerular basement membrane (GBM) and the epithelial filtration slits as the barrier. However, the prevailing point of view at present is that the slit diaphragms bridging the filtration slits are responsible for this important function, and evidence implicating the GBM is largely ignored or forgotten. In this issue of the JCI, Jarad et al. show that in laminin $\beta 2$-deficient (Lamb2-/-) mice, proteinuria can be directly attributed to the altered composition of the GBM (see the related article beginning on page 2272). Changes in the permeability of the GBM and its organization were primary to changes in the epithelium, as they preceded foot process effacement and loss of slit diaphragms.
\end{abstract}

It has been more than 50 years since the early days of EM, when the ultrastructural organization of the glomerular capillary wall was defined. Yet there is still no consensus concerning which component - the slit diaphragms bridging the filtration slits or the glomerular basement membrane $(\mathrm{GBM})$ - represents the primary glomerular filtration barrier. Over the intervening years, the pendulum has swung back and forth according to the interpretation of the evidence available. Since the discovery in 1999 of nephrin, a slit diaphragm protein, and its identification as the defective gene in patients with congenital nephrosis of the Finnish type (CNF) (1), the prevailing view has been that the slit diaphragms located in the filtration slits that attach the adjoining foot processes to one another contain

Nonstandard abbreviations used: GBM, glomerular basement membrane; Lamb2, laminin $\beta 2$.

Conflict of interest: The author has declared that no conflict of interest exists.

Citation for this article: J. Clin. Invest. 116:2090-2093 (2006). doi:10.1172/JCI29488. pores responsible for the molecular sieving that prevents passage of albumin across the capillary wall. The work of Jarad et al. (2) in this issue of the JCI clearly demonstrates that defects in the composition and integrity of the GBM meshwork can lead to proteinuria, thus emphasizing the crucial role of the GBM in filtration and lending support to the concept that the fibrillar meshwork of the GBM functions as the molecular sieve that retains albumin.

\section{The swinging of the pendulum}

The first direct experimental evidence aimed at identification of the glomerular filtration barrier was based on the use of the electron-dense tracer ferritin $(3,4)$ and later, graded dextrans of varying sizes (MW, 32,000-125,000 Da) (5), which pinpointed the GBM as the layer that prevents passage of both of these tracers. Ferritin and dextrans of the size of albumin or greater accumulated against the luminal surface of the GBM. Later on, quite a number of different tracers were used, and most accumulated against the GBM rather than in the epithelial slits. This work was superseded by findings obtained largely by Karnovsky and coworkers using enzymatic tracers of different sizes such as catalase (MW, 220,000 $\mathrm{Da}$ ) and HRP (MW, 40,000 Da), which were detected by a histochemical reaction (reviewed in ref. 6). These studies suffered from serious technical limitations (6), but they were interpreted as demonstrating that the filtration slits form the selective filter. Based on these studies, it was proposed that the GBM is a coarse pre-filter and the epithelial slits function as the crucial molecular sieving layer. This idea was reinforced by the discovery of a periodic structure interpreted as "slit-pores" in the slit membranes bridging the filtration slits (7). Later, Ryan and Karnovsky (8) observed that when renal blood flow was normal, plasma albumin, like ferritin and dextrans, was absent from the GBM and urinary spaces, but large amounts could be detected by immunocytochemistry in both sites if the tissue was immersion fixed or renal blood flow was interrupted by ligation of the renal artery. These studies underlined the importance of the GBM as the protein filter under normal flow conditions and helped to explain some of the authors' earlier results that had been obtained from kidney tissue fixed by immersion. Yet the view that the GBM is a coarse pre-filter and the epithelial slits serve as a fine filter restricting the passage of albumin has lived on and remained predominant in the field.

Based on the work of Brenner and associates on the importance of charge as a determinant of glomerular filtration, interest in the 1970s and 1980s shifted to defining the charge barrier (9). A sialic acid-rich, highly negatively charged cell-surface coat was found on podocytes, and regularly spaced, 